\title{
Preparation and characterization of Poly (D,L-lactide-co-glycolide) (PLGA) nanoparticles loaded with linamarin for controlled drug release
}

\begin{abstract}
Poly (D,L-lactide-co-glycolide) nanoparticles loaded with linamarin as a model drug were successfully prepared using the double emulsion solvent evaporation technique. The physicochemical characterization of the formulated nanoparticles revealed that they were spherical, nonaggregated, and negatively charged, with good drug encapsulation efficiencies $(>50 \%)$ and average particle sizes $<200 \mathrm{~nm}$. Interestingly, all the nanoparticles exhibited dibasic release profiles with a starting burst release within the first $8 \mathrm{~h}$, followed by a controlled release phase lasting four days. Thus, linamarin-loaded nanoparticles indicate a promising candidate for controlled drug release applications.
\end{abstract}

Keyword: Biodegradable; Controlled release; Linamarin; Nanoparticles; PLGA polymer 